\title{
Long non-coding RNA H19 is associated with polycystic ovary syndrome in Chinese women: a preliminary study
}

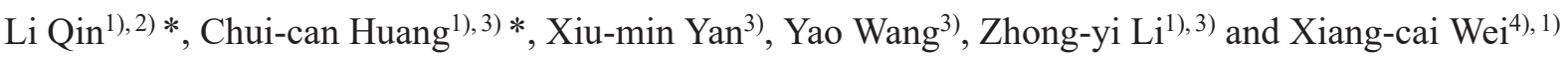 \\ 1) Department of Obstetrics and Gynecology, the First Clinical Medical College of Jinan University, Guangzhou, Guangdong 510632, \\ China \\ 2) Center for Reproductive Medicine, the Affiliated Hospital of Youjiang Medical University for Nationalities, Baise, Guangxi 533000, \\ China \\ 3) Department of Epidemiology, School of Medicine, Jinan University, Guangzhou, Guangdong 510632, China \\ 4) Guangdong Women and Children Hospital, Guangzhou, Guangdong 510000, China
}

\begin{abstract}
Polycystic ovary syndrome (PCOS) represents a serious reproductive and endocrine condition and is associated with high incidence rates. H19 is a compelling long noncoding RNA (lncRNA) which carries out a range of biological functions. However, prior to this study, little was known as to whether there was an association between lncRNA H19 and PCOS. In the current study, we used quantitative real-time polymerase chain reaction (qRT-PCR) to determine lncRNA H19 expression levels in peripheral blood leukocytes from patients with PCOS and compared this data with that derived from normal controls. We also screened data for potential relationships between lncRNA H19 and a range of endocrine variables in PCOS. The expression of lncRNA H19 was significantly higher in cases of PCOS than in controls. Individuals exhibiting higher expression levels of IncRNA H19 were associated with a significantly higher risk of PCOS than those with lower expression levels. Moreover, lncRNA H19 expression was positively correlated with fasting plasma glucose levels; this was the case with both raw data, and after adjustment for age and BMI in the PCOS group. However, lncRNA H19 expression showed no significant correlation with total testosterone or insulin resistance in either PCOS cases or the controls. In conclusion, we demonstrate the first evidence to indicate that lncRNA H19 is associated with PCOS, suggesting that elevated lncRNA H19 levels are a risk factor for PCOS. For susceptible individuals, lncRNA H19 may represent a useful biomarker of the early stages of endocrine and metabolic disorders in PCOS.
\end{abstract}

Key words: LncRNA, H19, Polycystic ovary syndrome, Fasting plasma glucose, Metabolic disorders

POLYCYSTIC OVARY SYNDROME (PCOS) is a gynecological endocrinopathy and represents one of the most common heterogeneous endocrine and metabolic disorders. Global data indicate that PCOS affects approximately $10 \%$ of women during their reproductive years [1]. In China, almost $5.6 \%$ women of childbearing age suffer from PCOS [2]. Worryingly, PCOS is a common cause of female infertility, accounting for $75 \%$ of cases involving anovulatory infertility [3]. PCOS is associated with a wide range of clinical manifestations, including metabolic, psychological and reproductive anomalies; previous research has shown that androgenic mechanisms play the predominant role in the PCOS phenotype

Submitted Jan. 9, 2019; Accepted Mar. 6, 2019 as EJ19-0004

Released online in J-STAGE as advance publication Apr. 13, 2019

Correspondence to: Xiang-cai Wei, PhD, Guangdong Women and Children Hospital, No. 521, Xingnandadao, Guangzhou Guangdong Province 510000, China.

E-mail: Dxcwei@163.com

*Contributed equally to this work
[4]. The typical clinical presentation of PCOS generally includes hyperandrogenism (clinical and/or biochemical) and menstrual irregularities; ultrasonography usually shows the presence of polycystic ovaries $[5,6]$. Furthermore, PCOS has been shown to exhibit strong association with insulin resistance (IR), dyslipidemia and obesity, and an increased risk of cardiovascular problems and diabetes. Consequently, PCOS can be considered as not just a disorder of the reproductive system but also a metabolic syndrome. Metabolic irregularities and endocrine sequelae can affect women across their lifetime [7]. However, the etiology and pathophysiology of PCOS remains poorly understood.

The identification of non-coding RNAs (ncRNAs) has opened up new avenues with which to explore the rapidly evolving field of PCOS. These are referred to as RNAs without coding potential and can be divided into long ncRNAs (lncRNAs) and small ncRNAs (microRNAs, piRNA and snoRNA). LncRNAs are non-coding RNAs which are more than 200 nucleotides in length and 
are found in a diverse array of species [8-10]. Functionally, IncRNAs represent a diverse class of transcripts, and can regulate gene expression at many different levels. Previous work has shown that abnormalities in the regulation of lncRNA expression was linked with a wide range of human diseases and disorders. Accumulating evidence now supports the pivotal role of IncRNAs in disease development and progression, incuding endocrine disorders, tumors and even metastasis [11-13]. In a previous study, Huang et al. [14] described the significant up- or downregulation $(>/=2$-fold change) of 623 lncRNAs and 260 messenger RNAs (mRNAs) in patients with PCOS, and claimed that such differences could be used to differentiate cumulus cells from patients with PCOS from those of normal controls. In another study, Liu et al. $[15,16]$ demonstrated the differential expression of C-Terminal binding protein 1 antisense (CTBP1AS) and lncRNA steroid receptor RNA activator (SRA) in patients with PCOS compared to controls, and stated that such findings might represent a risk factor for PCOS. Collectively, these data suggested that the diverse role of lncRNAs in the regulation of gene expression might provide some clues with which to explain the clinical heterogeneity of PCOS.

The first lncRNA to be discovered was H19 [17], with a molecular size of 2.6 kilobases. Research has shown that $\mathrm{H} 19$ is subject to genomic imprinting, along with the IGF2 gene, which is located adjacent to $\mathrm{H} 19$ at position 11 p15.5 [18]. It therefore follows that studying this locus, and the gene regulatory mechanisms that operate at this locus, might serve as a useful model with which to enhance our understanding of the molecular mechanisms involved in regulating gene expression. Owing to its specialized structure, lncRNA H19 is able to coordinate the functional role of a wide variety of transcription factors and coregulators, and may therefore act as a molecular platform to facilitate protein interactions [18]. Such proteins are involved in a wide array of physiological and pathological processes, including gene transcription, RNA metabolism, skeletal muscle differentiation, epigenetic modification and tumor development $[19,20]$; it is therefore very apparent that H19 has many functional roles. An accumulating body of evidence now supports the diverse mechanistic roles of IncRNA H19 in pancreatic cancer [21], prostate tumors [22], endometriosis [23] and infertility $[24,25]$. However, prior to this study, it was not known whether there was an association between lncRNA H19 and PCOS.

The objective of the present study was to identify potential links between lncRNA H19 and PCOS. Because PCOS represents a systemic endocrinopathy of the entire body and peripheral blood samples are very appropriate for gene expression studies [26], we deter- mined the expression levels of H19 in peripheral blood leukocytes from PCOS patients and compared this data to that derived from normal controls. We also attempted to correlate the expression of H19 with a range of key clinical parameters in PCOS patients. This study represents novel step forward in our attempts to understand the potential link between lncRNAs and the PCOS phenotype.

\section{Materials and Methods}

\section{Subjects}

In this study, we recruited 20 patients with PCOS and 20 matched healthy women from the gynecological reproductive clinic at Guangdong Women and Children Hospital. PCOS was diagnosed in accordance with the 2003 Rotterdam criteria, which required the presence of two or more of the following features: clinical or biochemical evidence of hyperandrogenism; oligoanovulation (oligomenorrhoea or amenorrhea), and polycystic ovaries upon ultrasonography (at least 12 follicles measuring 2-9 $\mathrm{mm}$ or an ovarian volume $>10 \mathrm{~cm}^{3}$ ). Patients were excluded from the study if they had congenital adrenal hyperplasia, Cushing's syndrome, androgen-secreting tumors or thyroid dysfunction. As a control, we recruited a group of matched healthy women in which there was no history of menstrual cycle problems, PCOS-related endocrine disorders or hyperandrogenism. None of the subjects recruited into this study had been administered with endocrine medicine for least three months prior to the study commencing. This study was approved by the hospital ethics committee and written informed consent was taken from each patient prior to recruitment.

\section{Data collection}

Physical examinations were carried out on all patients and a range of variables collected, including body weight, height, hip and waist circumference. We also determined modified Ferriman-Gallwey scores for each patient and determined body mass index (BMI, $\mathrm{kg} / \mathrm{m}^{2}$ ). All patients also received transvaginal ultrasonography.

Blood samples were collected between 8 am and 10 am following an overnight $(12 \mathrm{~h})$ period of fasting during either the follicular phase (days 2-5) of a natural menstrual cycle or during a progesterone-induced withdrawal bleed. These blood samples were used to obtain serum and determine the following parameters: luteinizing hormone (LH); follicle stimulating hormone (FSH); progesterone (P); estradiol (E2); prolactin (PRL); testosterone (T); inhibin B (INHB); anti-Mullerian hormone $(\mathrm{AMH})$; fasting insulin (FINS) and fasting plasma glucose (FPG). We also assessed Homeostasis model assess- 
ment: insulin resistance (HOMA-IR). It was determined as follows: [fasting plasma glucose $(\mathrm{mmol} / \mathrm{L}) \times$ fasting insulin $(\mathrm{lU} / \mathrm{mL})] / 22.5$. The oral glucose tolerance test (OGTT) was used to measure glucose levels over time and thus determine impaired glucose tolerance (IGT), impaired fasting glucose (IFG) and type 2 diabetes mellitus (T2DM); the specific criteria used were in accordance with those established by the World Health Organization.

\section{Isolation of peripheral blood leukocytes}

We obtained blood samples in EDTA tubes from patients with PCOS and from healthy controls. For each subject, approximately $5 \mathrm{~mL}$ of blood was diluted with an equal volume of phosphate-buffered saline (PBS), and then overlaid onto a Ficoll-Paque Plus kit (1:1 ratio). Each sample was then centrifuged at $800 \mathrm{~g}$ for 30 minutes at $4^{\circ} \mathrm{C}$. After centrifugation, the middle layer, containing the mononuclear cells, was obtained and washed twice with PBS to remove Ficoll and plasma. Samples were then stored at $-80^{\circ} \mathrm{C}$ to await further experiments.

\section{$R N A$ isolation and RT-PCR}

Total RNA was extracted from isolated peripheral blood leukocytes using the RNeasy Mini kit (QIAGEN, Hilden, Germany) in accordance with the manufacturer's guidelines. The concentration and purity of the resultant RNA was then determined using an ND-1000 NanoDrop spectrophotometer (NanoDrop, Wilmington, Delaware) and by denaturing gel electrophoresis, respectively. Next, RNA was reverse-transcribed into complementary DNA (cDNA) using PrimeScript ${ }^{\mathrm{TM}}$ RT Master Mix (Perfect Real Time) Reagent Kit (TaKaRa, Japan) in a Thermal Cycler Dice ${ }^{\mathrm{TM}}$ Real Time PCR System (Bio-Rad Research) using the following cycle: 15 minutes at $37^{\circ} \mathrm{C}$ for reverse transcription, 5 seconds at $85^{\circ} \mathrm{C}$ to inactivate the reverse transcriptase, and cold-storage at $4^{\circ} \mathrm{C}$.

\section{Quantitative real-time polymerase chain reaction}

For each subject, the expression of H19 was quantified by quantitative real-time polymerase chain reaction (qRT-PCR) using the SYBR ${ }^{\circledR}$ Premix Ex Taq ${ }^{\mathrm{TM}}$ (Takara) in accordance with the manufacturer's guidelines. All reactions were carried out in a Thermal Cycler Dice ${ }^{\mathrm{TM}}$ Real Time System II -CFX96 Real-time PCR system (Bio-Rad Research) with the following cycling conditions: 30 seconds at $95^{\circ} \mathrm{C}$ for pre-denaturation and PCR amplification for 5 seconds at $95^{\circ} \mathrm{C}$ ( 45 cycles) followed by primer annealing for 30 seconds at $60^{\circ} \mathrm{C}$. Melt curve analysis was used to determine the specificity of the reactions. Agarose gel electrophoresis was then used to evaluate the molecualr weight of the resultant PCR prod- ucts. H19 expression levels were then evaluated using the comparative threshold $(\mathrm{Ct})$ cycle method relative to the expression levels of glyceraldehyde-3-phosphate dehydrogenase (GAPDH) as a control. PCR reactions utilized the following primers for lncRNA H19: 5'AACGATGTGTGTGCCTTGAT-3' (forward) and 5'TGGTCCGACAGGGTGAATT-3' (reverse). GAPDH RNA was amplified using the following primers: 5'TGACTTCAACAGCGACACCCA-3' (forward) and 5'CACCCTGTTGCTGTAGCCAAA-3' (reverse). Each set of qRT-PCR reactions was repeated in triplicate.

\section{Statistical analysis}

All statistical analyses were undertaken using SPSS 18.0 statistical software. Data are presented as means \pm standard error of the mean (SEM) or median (with interquartile range). Normally distributed continuous variables were evaluated using the Shapiro-Wilk test. Differences in specific parameters between PCOS patients and healthy controls were compared using either the Student's $t$ test for quantitative data (if data followed a Gaussian distribution) or the Mann-Whitney U test for data which did not follow the Gaussian distribution. For PCOS patients and controls, we divided H19 expression into binary groups using limits imposed by the control group: levels $<0.1706$ were defined as low expression while levels $\geq 0.1706$ were defined as high expression. Separate Pearson or Spearman's rank correlation coefficients were used to search for linear relationships between H19 expression and specific clinical/biochemical parameters. In order to explore the potential effect of H19 expression on FPG, we constructed a multiple linear repression model which featured FPG as a dependent variable and age, H19 expression and BMI as independent variables. Data were considered to be statistically significant when $p<0.05$.

\section{Results}

\section{Clinical data}

Table 1 shows clinical, metabolic and hormonal data from our PCOS patients and healthy controls. No significant differences were detected between the two groups of subjects in terms of age. In comparison to normal controls, PCOS patients exhibited significantly higher levels of BMI, T, LH, LH/FSH, AMH, FINS, FPG, and HOMA-IR $(p<0.05)$. After adjustment for BMI, significant differences were still evident between patients and controls for T, AMH, FINS, FPG and HOMA-IR.

\section{LncRNA H19 expression in PCOS patients and normal controls}

Data shown in Fig. 1 indicates that mean lncRNA H19 
Table 1 Clinical characteristic of controls and PCOS patients

\begin{tabular}{lcccc}
\hline Variables & Controls & PCOS patients & $p$ value & $p$ value (BMI-adjusted) \\
\hline Number & 20 & 20 & & \\
\hline Age $($ years $)$ & $31.7 \pm 1.52$ & $28.45 \pm 1.35$ & 0.118 & 0.551 \\
\hline BMI $\left(\mathrm{kg} / \mathrm{m}^{2}\right)$ & $20.56 \pm 0.58$ & $25.21 \pm 0.86$ & $\mathbf{0 . 0 0 1}$ & \\
\hline T $(\mathrm{nmol} / \mathrm{L})$ & $1.02 \pm 0.07$ & $1.74 \pm 0.11$ & $<\mathbf{0 . 0 0 1}$ & $\mathbf{0 . 0 0 8}$ \\
\hline E2 $(\mathrm{pmmol} / \mathrm{L})$ & $154.05 \pm 11.08$ & $155.13 \pm 14.51$ & 0.953 & 0.896 \\
\hline P $(\mathrm{nmol} / \mathrm{L})$ & $1.22 \pm 0.13$ & $1.06 \pm 0.09$ & 0.304 & 0.626 \\
\hline FSH $(\mathrm{mU} / \mathrm{mL})$ & $5.96 \pm 0.28$ & $5.22 \pm 0.25$ & 0.058 & 0.333 \\
\hline LH $(\mathrm{mU} / \mathrm{mL})$ & $3.75 \pm 0.39$ & $5.68 \pm 0.60$ & $\mathbf{0 . 0 1 1}$ & 0.187 \\
\hline PRL $(\mathrm{ng} / \mathrm{mL})$ & $322.95 \pm 38.23$ & $340.29 \pm 50.52$ & 0.786 & 0.469 \\
\hline LH/FSH & $0.66 \pm 0.08$ & $1.11 \pm 0.11$ & $\mathbf{0 . 0 0 2}$ & 0.549 \\
\hline AMH $(\mathrm{ng} / \mathrm{mL})$ & $5.00 \pm 0.69$ & $9.84 \pm 1.24$ & $\mathbf{0 . 0 0 2}$ & $\mathbf{0 . 0 4 5}$ \\
\hline INHB $(\mathrm{pg} / \mathrm{mL})$ & $116.94 \pm 11.74$ & $96.36 \pm 15.95$ & 0.305 & 0.850 \\
\hline FINS $(\mathrm{uU} / \mathrm{mL})$ & $9.10 \pm 0.99$ & $17.69 \pm 2.37$ & $\mathbf{0 . 0 0 2}$ & $\mathbf{0 . 0 3 0}$ \\
\hline FPG $(\mathrm{mmol} / \mathrm{L})$ & $4.91 \pm 0.13$ & $5.64 \pm 0.21$ & $\mathbf{0 . 0 0 5}$ & $\mathbf{0 . 0 0 0}$ \\
\hline HOMAIR & $1.96 \pm 0.20$ & $4.58 \pm 0.73$ & $\mathbf{0 . 0 0 1}$ & $\mathbf{0 . 0 0 4}$ \\
\hline HOMA $\beta$ & $169.31 \pm 33.81$ & $185.08 \pm 27.96$ & 0.721 & 0.545 \\
\hline
\end{tabular}

Abbreviations: BMI, body mass index; T, testosterone; E2, estradiol; P, Progesterone; FSH, folliclestimulating hormone; LH, luteinizing hormone; PRL, prolactin; AMH, anti-Mullerian hormone; INHB, inhibin B; FINS, fasting insulins; FPG, fasting plasma glucose; HOMA-IR, homeostasis model assessment: insulin resistance. SEM, standard error of the mean. Values are expressed as mean $\pm \mathrm{SEM}$ or median (interquartile range). $p$ values were obtained from unpaired two-tailed Student's $t$-test or Mann-Whitney U test. Clinical indexes with significant differences $(p<0.05)$ are in bold.

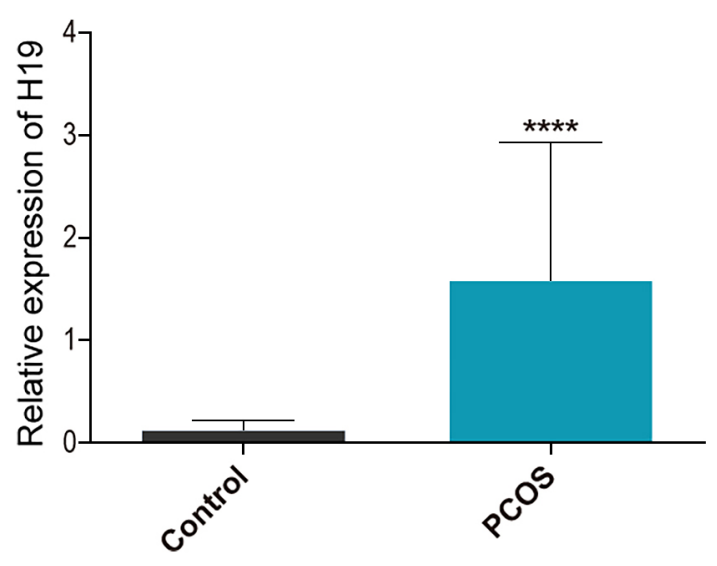

Fig. 1 The expression profile of IncRNA H19 as measured in the peripheral blood of PCOS patients and controls by quantitative real-time polymerase chain reaction (qRTPCR). ${ }^{*} p<0.05 v s$. the control group.

expression levels in PCOS patients was significantly higher than in normal controls $(1.59 \pm 0.30$ vs. 0.13 $\pm 0.02, p<0.001)$ after adjustment for both BMI and age. Similar results were obtained for each of the triplicate evaluations performed.

\section{Links between IncRNA H19 expression and PCOS}

Next, we aimed to identify potential relationships between H19 expression and the risk of PCOS. Table 2 shows that individuals with higher levels of H19 expression had a significantly greater risk of disease than those with lower levels of expression (odds ratio $=27.87,95 \%$ confidence interval $=2.571-301.499, p=0.006$ ) after adjustment for BMI.

\section{Correlations between IncRNA H19 expression and PCOS parameters}

Separate Pearson or Spearman's rank correlation coefficients were determined to investigate for potential relationships between H19 expression and specific clinical/ biochemical parameters. In these analyses, H19 was used as a dependent variable, while clinical parameters (from both PCOS patients and controls) were used as independent variables. Table 3 shows that there was a significant positive correlation between H19 expression and FPG only in the PCOS group $(r=0.709, p=0.001)$ following adjustment for BMI and age. There was no such relationship between H19 expression and T, HOMA-IR or any of the other clinical characteristics. 
Table 2 Odds ratio of PCOS events by expression of lncRNA H19

\begin{tabular}{|c|c|c|c|c|c|c|c|}
\hline & \multicolumn{2}{|c|}{ Controls $(n=20)$} & \multicolumn{2}{|c|}{ PCOS patients $(n=20)$} & \multirow{2}{*}{$O R$} & \multirow{2}{*}{$95 \% C I$} & \multirow{2}{*}{$p$ value } \\
\hline & Low & High & Low & High & & & \\
\hline No adjustment & $15(75 \%)$ & $5(25 \%)$ & $1(5 \%)$ & $19(95 \%)$ & 57 & $6.0-541.466$ & 0.000 \\
\hline Adjusted for BMI & & & & & 27.87 & $2.571-301.499$ & 0.006 \\
\hline
\end{tabular}

Abbreviations: BMI, body mass index; PCOS, polycystic ovary syndrome; $O R$, odds ratio; $95 \% C I$, confidence intervals.

a Data are $n(\%)$, unless otherwise indicated. LncRNA H19 expression for binary groups cutoffs were $<0.1706$ for the low expression, $\geq 0.1706$ for the high expression. The $O R, 95 \% C I$, and $p$ value were estimated for PCOS events in the high expression group compared to the low expression using logistic regression models. $p$ values $<0.05$ are in bold.

Table 3 Correlation between the expression of lncRNA H19 and subjects' clinical parameters ${ }^{\mathrm{a}}$

\begin{tabular}{|c|c|c|c|c|}
\hline \multirow[t]{2}{*}{ Variables } & \multicolumn{2}{|c|}{ Controls } & \multicolumn{2}{|c|}{ PCOS patients } \\
\hline & $r$ & $p$ & $r$ & $p$ \\
\hline \multicolumn{5}{|l|}{ BMI $\left(\mathrm{kg} / \mathrm{m}^{2}\right)$} \\
\hline \multicolumn{5}{|l|}{ Age (years) } \\
\hline $\mathrm{T}(\mathrm{nmol} / \mathrm{L})$ & -0.196 & 0.523 & 0.177 & 0.482 \\
\hline E2 (pmmol/L) & -0.152 & 0.584 & -0.082 & 0.748 \\
\hline $\mathrm{P}(\mathrm{nmol} / \mathrm{L})$ & 0.05 & 0.843 & 0.102 & 0.688 \\
\hline FSH (mU/mL) & -0.009 & 0.973 & 0.448 & 0.055 \\
\hline LH (mU/mL) & 0.438 & 0.061 & 0.239 & 0.339 \\
\hline PRL (ng/mL) & -0.368 & 0.133 & 0.108 & 0.670 \\
\hline LH/FSH & 0.406 & 0.095 & -0.070 & 0.784 \\
\hline AMH (ng/mL) & 0.192 & 0.446 & 0.215 & 0.393 \\
\hline INHB (pg/mL) & -0.186 & 0.460 & -0.141 & 0.577 \\
\hline FINS (uU/mL) & 0.086 & 0.620 & 0.25 & 0.318 \\
\hline FPG (mmol/L) & 0.156 & 0.536 & 0.709 & 0.001 \\
\hline HOMAIR & -0.015 & 0.951 & 0.397 & 0.103 \\
\hline НОМА $\beta$ & -0.054 & 0.831 & -0.243 & 0.331 \\
\hline
\end{tabular}

a The correlation coefficient $(r)$ and $p$ value were adjusted for age and BMI. $p$ values $<0.05$ are in bold. For abbreviations refer Table 1.

\section{The Effects of H19 Expression upon FPG}

Table 4 shows a positive correlation between H19 expression and FPG both for raw data and data that had been adjusted for BMI and age. We constructed a model which used H19 expression, BMI and age as predictors of PCOS risk and found that this explained $46.3 \%$ (adjusted $\left.R^{2}\right)$ of the variability in FPG levels $(p=0.001)$. Similar analysis, carried out on the normal controls, failed to identify any statistically significant differences in any of the biochemical parameters tested (data not shown).

\section{Discussion}

The present study investigated lncRNA H19 expression in peripheral blood leukocytes of patients with
PCOS and healthy matched controls. We believe that this is the first study to evaluate the association between IncRNA H19 and PCOS. Women with PCOS showed significantly higher lncRNA H19 expression levels than healthy controls; this was also the case when potential confounding factors, such as age and BMI, were controlled for. We also found that females with higher expression levels of lncRNA H19 had a significantly greater risk of disease than those with lower expression levels after adjusting for BMI. This indicated that IncRNA H19 may contribute to the etiology of PCOS, at least in part.

LncRNA H19 has been proven to play a key role in the development of a range of diseases [21-25]. A growing body of evidence now implicates lncRNA H19 in a wide array of physiological and pathological processes, including gene transcription, RNA metabolism, differen- 
Table 4 Multiple linear regression models with FPG as the dependent variable in PCOS

\begin{tabular}{lcc}
\hline & Standardized $\beta$ coefficient & $p$ value \\
\hline Unadjusted model $\left(R^{2}=0.376\right)$ & & \\
$H 19$ expression & 0.594 & $\mathbf{0 . 0 0 6}$ \\
Adjusted model $\left(R^{2}=0.463\right)$ & & \\
H19 expression & 0.660 & $\mathbf{0 . 0 0 1}$ \\
Age & 0.377 & \\
BMI & -0.256 & \\
\hline
\end{tabular}

${ }^{\mathrm{a}}$ The $p$ values $<0.05$ are in bold.

tiation, epigenetic modification, and the development of tumors $[19,20]$. One previous study reported that H19 expression was downregulated in pancreatic islets, and may therefore represent a potential mechanism for the impairment of islet function and ultrastructure [27]. Another research showed that lncRNA H19 was implicated in the pathogenesis of neuroendocrine prostate cancer, an androgen-dependent, thus, indicating that lncRNA H19 appears to play a role in the regulation of androgen levels [28]. Evidence also supports the role of lncRNA H19 in a range of biological processes; we hypothesized that lncRNA H19 may be associated with the etiology of PCOS [1]. Our current data showed that H19 expression was significantly up-regulated in PCOS patients and that only very low levels of expression were evident in normal controls. These novel observations indicated that lncRNA H19 may play a key role in the occurrence and development of PCOS.

Existing data show that there is a strong association between PCOS and metabolic syndrome (MetS) [29]. Indeed, PCOS patients appear to have higher risk levels of developing type 2 diabetes and cardiovascular disease [30], predominantly due to the incidence of various endocrine and metabolic disorders, including insulin resistance, hyperandrogenemia, obesity, hyperinsulinemia, hypertension and hyperlipidemia. The pathogenesis of PCOS has yet to be fully elucidated; this is largely due to the fact that the clinical manifestations of PCOS are very heterogeneous. The two most typical manifestations of PCOS are hyperandrogenism and insulin resistance. Indeed, the majority of PCOS patients exhibit insulin resistance. Due to the fact that hyperinsulinemia, insulin resistance and high plasma glucose are closely related, it follows that the determination of glycemic status may represent a useful indicator of insulin resistance in patients with PCOS.

Our current data clear showed that FPG, FINS and HOMA-IR were significantly higher in PCOS patients than in normal controls (Table 1). Further analyses indicated that the expression levels of H19 were associated with a linear increase in FPG only in patients with PCOS. We also created a multiple linear regression model and revealed a positive and significant correlation between lncRNA H19 and FPG in patients with PCOS ( $r$ $=0.709, p=0.001$ ) following adjustment for BMI and age. FPG is known to be the most frequent and sensitive indicator for detecting the early stages of several metabolic diseases and is also known to represent a stronger predictor for type 2 diabetes than TG/HDL-C, TyG and HOMA-IR indices [31]. Previous research showed that FPG was most strongly associated with incident diabetes, followed by $2 \mathrm{hPG}, \mathrm{HbA} 1 \mathrm{c}, \mathrm{HOMA}-\mathrm{IR}$ and fasting insulin [32]. Indeed, pre-stimulatory glucose levels have been shown to influence both the function of $\beta$-cells and insulin sensitivity in very different ways when compared between non-diabetics and those with type 2 diabetes [33]. Furthermore, it is clear that the impairment of $\beta$ cell function and the occurrence of increased insulin resistance become evident prior to the development of metabolic syndrome, IGT or IFG. Indeed, the function of $\beta$-cells and insulin sensitivity become worse in direct proportion with the number of traits that are associated with metabolic, thus increasing glucose levels [34]. Furthermore, it is well known that insulin resistance plays a key role in the pathogenesis and maintenance of PCOS, and that PCOS patients are at higher risk of type 2 diabetes mellitus. In the present study, we revealed that FPG was significantly higher in patients with PCOS than in the controls. Subsequent oral glucose tolerance test (OGTT) showed that some of these patients had IGT or IFG, but had not been diagnosed with diabetes at that time. We also found that FPG was positively associated with lncRNA H19 expression levels in PCOS patients and that this relationship did not exist in the controls. Since FPG is a sensitive indicator for the early stages of several metabolic diseases, and because PCOS is strongly related to metabolic syndrome, we hypothesized that higher expression levels of IncRNA H19 may be a key factor in the early stages of metabolic disorders in PCOS patients. Further studies are now required to spe- 
cifically address this hypothesis.

Hyperandrogenism is another predominant feature of PCOS. Several factors support the fact that hyperandrogenism can determine the characteristic phenotype PCOS, including, abnormalities of the menstrual cycle, hirsutism and polycystic ovaries [35-37]. Excessive levels of androgens may also promote insulin resistance and the dysfunction of $\beta$-cells in PCOS patients [38]. One previous study showed implicated lncRNA H19 in the development of neuroendocrine prostate cancer (NEPC), an androgen-related disease; this was suggestive of a potential regulatory role for lncRNA H19 expression with androgen levels [28]. However, in the present study, we found no evidence of a relationship between lncRNA H19 expression and either TT or HOMA-IR in either PCOS patients or the controls. Although PCOS patients show higher TT, FINS and HOMA-IR relative to controls, there was no independent relationship between testosterone and insulin in terms of the expression of IncRNA H19. Consequently, elevated levels of lncRNA H19 in peripheral blood does not play a key role in either hyperinsulinemia or hyperandrogenism. However, we did identify significant up-regulation of lncRNA H19 expression in patients with PCOS, and a significant reduction of IncRNA H19 expression in the controls (Fig. 1), suggesting that low levels of lncRNA H19 may act in a protective capacity and prevent the onset of PCOS. In contrast, individuals with higher levels of lncRNA H19 had a significantly higher risk of disease relative to those with lower expression levels (Table 2). Future studies should investigate the mechanisms underlying these observations. Consequently, we hypothesize that elevated H19 expression may be associated with the pathogenesis of PCOS by some other, currently unknown, mechanism, such as genetic imprinting, rather than simply an excess of androgens.

It is worth highlighting that $\mathrm{H} 19$ gene is an imprinted gene, and is localized on its chromosome adjacent to insulin-like growth factor 2 (IGF2), another imprinted gene; however, H19 is expressed solely from the maternal allele, while IGF2 is expressed solely from the paternal allele. Previous research has shown that the H19/ IGF2 locus, both genes share a common set of enhancers, which are located downstream of the H19 gene [18]. Differentially methylated regions in these two genes have also been implicated in a range of biological processes and diseases, largely because of their role regulating composition of the body [39-42]; however, little research has been carried out in terms of PCOS in this respect. One previous study showed that IGF2 is a host gene of miR-483-5p and recorded significantly lower levels of IGF2 in the cumulus cells of women with PCOS [43]. This may provide important clues for future research aiming to identify the mechanisms underlying PCOS. Other research has shown that obesity is linked to the pathogenesis of PCOS [44]. Indeed, adiposity is known to influence the severity of PCOS in terms of both clinical and endocrine features. Research has shown that excessive levels of body fat, particularly intraabdominal fat, is a leading risk factor for the development of metabolic diseases. Furthermore, methylation levels of DMR regions in the IGF2 gene in leukocytes have been associated with total body fat content and the distribution of fat in the viscera and liver in manner that was independent of total adiposity [45]. These data are indicative of a potential association between IGF2 and PCOS. While the expression profile of IGF2 was beyond the remit of the current study, it is impossible to exclude a potential role for IGF2 in PCOS and further research is now required to investigate this further.

One issue to consider is that PCOS represents a systemic endocrinopathy. While peripheral blood samples represent a readily accessible source of material for analysis, and may indicate the overall state of the entire body at the point when the samples were collected, there are still critical limitations in the use of peripheral blood leukocytes. For example, the genetic analysis of peripheral blood leukocytes will not necessarily reflect the expression of H19 in specific target tissues such as the ovary. Additional research is now required to further validate the use of peripheral blood leukocytes when investigating gene expression in a tissue regulated by hormones. Another key limitation of the present study is our relatively small sample size. However, we believe that our group of PCOS patients were well suited for the investigations undertaken because the group was homogeneous. Furthermore, although our study only provided a limited amount of data related to lncRNA H19 expression, we did ascertain several significant relationships which will serve to provide novel insight into the potential mechanisms underlying PCOS.

In conclusion, we identified that the expression levels of lncRNA H19 are associated with PCOS and established the possibility that abnormal H19 expression may represent a risk factor for PCOS in the Chinese population. FPG was also found to be positively associated with the expression levels of IncRNA H19 in women with PCOS but not in controls, thus indicating that the elevated expression of IncRNA H19 may represent a key factor in the onset of endocrine and metabolic disorders in PCOS. However, while there is no definitive proof for a causal link between lncRNA H19 and PCOS, it is possible that IncRNA H19 may contribute to the development of PCOS, at least in part. Our future research will therefore aim to investigate the function of H19 in vivo and investigate the potential association between lncRNA 
H19 and PCOS in cell lines and other ethnicities. We believe that by identifying the signaling pathways which feature lncRNA H19, it will be possible to gain a better understanding of the pathophysiology underlying PCOS and identify novel targets for diagnosis and therapy.

\section{Acknowledgements}

This study was financially supported by the 2018 Natural Science Foundation of Baise, China (No. 20182508). Supported by the "139" medical high-level talent training plan and thousands of young and middleaged backbone teachers cultivation plan of Guangxi Province, China (No. 2019KY0573). Supported by the 2018-2020 Professional and experimental practice teaching base construction projects of Guangxi Province, China.

\section{Disclosure}

The authors declare that they have no competing interests.

\section{References}

1. Goodarzi MO, Dumesic DA, Chazenbalk G, Azziz R (2011) Polycystic ovary syndrome: etiology, pathogenesis and diagnosis. Nat Rev Endocrinol 7: 219-231.

2. Li R, Zhang Q, Yang D, Li S, Lu S, et al. (2013) Prevalence of polycystic ovary syndrome in women in China: a large community-based study. Hum Reprod 28: 25622569.

3. Legro RS, Arslanian SA, Ehrmann DA, Hoeger KM, Murad MH, et al. (2013) Diagnosis and treatment of polycystic ovary syndrome: an Endocrine Society clinical practice guideline. J Clin Endocrinol Metab 98: 45654592.

4. Sung YA, Oh JY, Chung H, Lee H (2014) Hyperandrogenemia is implicated in both the metabolic and reproductive morbidities of polycystic ovary syndrome. Fertil Steril 101: 840-845.

5. Xia H, Zhang R, Sun X, Wang L, Zhang W (2017) Risk factors for preeclampsia in infertile Chinese women with polycystic ovary syndrome: a prospective cohort study. $J$ Clin Hypertens (Greenwich) 19: 504-509.

6. Reddy BM, Kommoju UJ, Dasgupta S, Rayabarapu P (2016) Association of type 2 diabetes mellitus genes in polycystic ovary syndrome aetiology among women from southern India. Indian J Med Res 144: 400-408.

7. Siklar Z, Berberoglu M, Camtosun E, Kocaay P (2015) Diagnostic characteristics and metabolic risk factors of cases with polycystic ovary syndrome during adolescence. J Pediatr Adolesc Gynecol 28: 78-83.

8. Jia H, Osak M, Bogu GK, Stanton LW, Johnson R, et al. (2010) Genome-wide computational identification and manual annotation of human long noncoding RNA genes. RNA 16: 1478-1487.

9. Derrien T, Johnson R, Bussotti G, Tanzer A, Djebali S, et al. (2012) The GENCODE v7 catalog of human long noncoding RNAs: analysis of their gene structure, evolution, and expression. Genome Res 22: 1775-1789.

10. Pauli A, Valen E, Lin MF, Garber M, Vastenhouw NL, et al. (2012) Systematic identification of long noncoding RNAs expressed during zebrafish embryogenesis. Genome Res 22: 577-591.

11. Xu Z, Yan Y, Qian L, Gong Z (2017) Long non-coding
RNAs act as regulators of cell autophagy in diseases (Review). Oncol Rep 37: 1359-1366.

12. Tang Y, Cheung BB, Atmadibrata B, Marshall GM, Dinger ME, et al. (2017) The regulatory role of long noncoding RNAs in cancer. Cancer Lett 391: 12-19.

13. Zuo L, Tan Y, Wang Z, Wang KS, Zhang X, et al. (2016) Long noncoding RNAs in psychiatric disorders. Psychiatr Genet 26: 109-116.

14. Huang X, Hao C, Bao H, Wang M, Dai H (2016) Aberrant expression of long noncoding RNAs in cumulus cells isolated from PCOS patients. $J$ Assist Reprod Genet 33: 111121.

15. Liu Z, Hao C, Huang X, Zhang N, Bao H, et al. (2015) Peripheral blood leukocyte expression level of lncRNA steroid receptor RNA activator (SRA) and its association with polycystic ovary syndrome: a case control study. Gynecol Endocrinol 31: 363-368.

16. Liu Z, Hao C, Song D, Zhang N, Bao H, et al. (2015) Androgen receptor coregulator CTBP1-AS is associated with polycystic ovary syndrome in Chinese women: a preliminary study. Reprod Sci 22: 829-837.

17. Brannan CI, Dees EC, Ingram RS, Tilghman SM (1990) The product of the H19 gene may function as an RNA. Mol Cell Biol 10: 28-36.

18. Angrand PO, Vennin C, Le Bourhis X, Adriaenssens E (2015) The role of long non-coding RNAs in genome formatting and expression. Front Genet 6: 165

19. Liu Y, Li G, Zhang JF (2017) The role of long non-coding RNA H19 in musculoskeletal system: a new player in an old game. Exp Cell Res 360: 61-65.

20. Zhang L, Zhou Y, Huang T, Cheng AS, Yu J, et al. (2017) The interplay of LncRNA-H19 and its binding partners in physiological process and gastric carcinogenesis. Int $\mathrm{J} \mathrm{Mol}$ Sci 18: pii: E450.

21. Yoshimura H, Matsuda Y, Yamamoto M, Michishita M, Takahashi K, et al. (2018) Reduced expression of the H19 long non-coding RNA inhibits pancreatic cancer metastasis. Lab Invest 98: 814-824.

22. Sun SC, Zhao H, Liu R, Wang BL, Liu YQ, et al. (2017) Expression of long non-coding RNA H19 in prostate cancer and its effect on the proliferation and glycometabolism 
of human prostate cancer cells. Zhonghua Nan Ke Xue 23: 120-124 (In Chinese).

23. Ghazal S, McKinnon B, Zhou J, Mueller M, Men Y, et al. (2015) H19 lncRNA alters stromal cell growth via IGF signaling in the endometrium of women with endometriosis. EMBO Mol Med 7: 996-1003.

24. Santi D, De Vincentis S, Magnani E, Spaggiari G (2017) Impairment of sperm DNA methylation in male infertility: a meta-analytic study. Andrology 5: 695-703.

25. Nasri F, Gharesi-Fard B, Namavar Jahromi B, Farazi-Fard MA, Banaei M, et al. (2017) Sperm DNA methylation of H19 imprinted gene and male infertility. Andrologia 49: e12766.

26. Oleszczak B, Szablewski L, Pliszka M, Gluszak O, Stopinska-Gluszak U (2014) Transport of deoxy-Dglucose into lymphocytes of patients with polycystic ovary syndrome. Endocrine 47: 618-624.

27. Ding GL, Wang FF, Shu J, Tian S, Jiang Y, et al. (2012) Transgenerational glucose intolerance with Igf2/H19 epigenetic alterations in mouse islet induced by intrauterine hyperglycemia. Diabetes 61: 1133-1142.

28. Ramnarine VR, Alshalalfa M, Mo F, Nabavi N, Erho N, et al. (2018) The long noncoding RNA landscape of neuroendocrine prostate cancer and its clinical implications. Gigascience 7: giy050.

29. Shah D, Rasool S (2016) Polycystic ovary syndrome and metabolic syndrome: the worrisome twosome? Climacteric 19: 7-16.

30. Kupreeva M, Diane A, Lehner R, Watts R, Ghosh M, et al. (2019) Effect of metformin and flutamide on insulin, lipogenic and androgen-estrogen signaling, and cardiometabolic risk in a PCOS-prone metabolic syndrome rodent model. Am J Physiol Endocrinol Metab 316: E16-E33.

31. Tohidi M, Baghbani-Oskouei A, Ahanchi NS, Azizi F, Hadaegh F (2018) Fasting plasma glucose is a stronger predictor of diabetes than triglyceride-glucose index, triglycerides/high-density lipoprotein cholesterol, and homeostasis model assessment of insulin resistance: Tehran Lipid and Glucose Study. Acta Diabetol 55: $1067-$ 1074.

32. Ruijgrok C, Dekker JM, Beulens JW, Brouwer IA, Coupe $\mathrm{VMH}$, et al. (2018) Size and shape of the associations of glucose, HbA1c, insulin and HOMA-IR with incident type 2 diabetes: the Hoorn Study. Diabetologia 61: 93-100.

33. Seghieri M, Rebelos E, Astiarraga BD, Baldi S, Mari A, et al. (2016) Impact of a mild decrease in fasting plasma glucose on beta-cell function in healthy subjects and patients with type 2 diabetes. Am J Physiol Endocrinol Metab 310:
E919-E924.

34. Cubeddu LX, Hoffmann IS (2012) Impact of traits of metabolic syndrome on beta-cell function and insulin resistance in normal fasting, normal glucose tolerant subjects. Metab Syndr Relat Disord 10: 344-350.

35. Schuring AN, Welp A, Gromoll J, Zitzmann M, Sonntag B, et al. (2012) Role of the CAG repeat polymorphism of the androgen receptor gene in polycystic ovary syndrome (PCOS). Exp Clin Endocrinol Diabetes 120: 73-79.

36. Dewailly D, Catteau-Jonard S, Reyss AC, Leroy M, Pigny P (2006) Oligoanovulation with polycystic ovaries but not overt hyperandrogenism. J Clin Endocrinol Metab 91: 3922-3927.

37. Azziz R, Woods KS, Reyna R, Key TJ, Knochenhauer ES, et al. (2004) The prevalence and features of the polycystic ovary syndrome in an unselected population. J Clin Endocrinol Metab 89: 2745-2749.

38. Zhang B, Wang J, Shen S, Liu J, Sun J, et al. (2018) Association of androgen excess with glucose intolerance in women with polycystic ovary syndrome. Biomed Res Int 2018: 6869705.

39. Coto E, Diaz Corte C, Tranche S, Gomez J, Reguero JR, et al. (2018) Genetic variation in the H19-IGF2 cluster might confer risk of developing impaired renal function. DNA Cell Biol 37: 617-625.

40. Lou H, Le F, Hu M, Yang X, Li L, et al. (2018) Aberrant DNA methylation of IGF2-H19 locus in human fetus and in spermatozoa from assisted reproductive technologies. Reprod Sci: 1933719118802052.

41. Park KS, Mitra A, Rahat B, Kim K, Pfeifer K (2017) Loss of imprinting mutations define both distinct and overlapping roles for misexpression of IGF2 and of H19 lncRNA. Nucleic Acids Res 45: 12766-12779.

42. Rotwein P (2018) Similarity and variation in the insulinlike growth factor 2-H19 locus in primates. Physiol Genomics 50: 425-439.

43. Shi L, Liu S, Zhao W, Shi J (2015) miR-483-5p and miR-486-5p are down-regulated in cumulus cells of metaphase II oocytes from women with polycystic ovary syndrome. Reprod Biomed Online 31: 565-572.

44. Moran C, Arriaga M, Rodriguez G, Moran S (2012) Obesity differentially affects phenotypes of polycystic ovary syndrome. Int J Endocrinol 2012: 317241.

45. Song MA, Ernst T, Tiirikainen M, Tost J, Wilkens LR, et al. (2018) Methylation of imprinted IGF2 regions is associated with total, visceral, and hepatic adiposity in postmenopausal women. Epigenetics 13: 858-865. 